\title{
Asymptotical mean-square stability of linear $\vartheta$-methods for stochastic pantograph differential equations: variable stepsize and transformation approach
}

\author{
xiaochen yang ${ }^{1}$, Zhanwen Yang $^{1}$, and Yu Xiao ${ }^{1}$ \\ ${ }^{1}$ Harbin Institute of Technology
}

May 23, 2020

\begin{abstract}
The paper deals with the asymptotical mean-square stability of the linear $\vartheta$-methods under variable stepsize and transformation approach for stochastic pantograph differential equations. A limiting equation for the analysis of numerical stability is introduced by Kronecker products. Under the condition which guarantee the stability of exact solutions, the optimal stability region of the linear $\vartheta$-methods under variable stepsize is given by using the limiting equation, i.e., $\vartheta[?]$ ( $1 / 2,1]$, which is the same to the deterministic problems. Moreover the linear $\vartheta$-methods under the transformation approach are also considered and the result of the stability is improved for $\vartheta=1 / 2$. Finally, numerical examples are given to illustrate the asymptotical meansquare stability under variable stepsize and transformation approach.
\end{abstract}

\section{Hosted file}

manuscript.pdf available at https://authorea.com/users/325577/articles/453565-asymptoticalmean-square-stability-of-linear-\%CE\%B8-methods-for-stochastic-pantograph-di $\% E F \% A C \%$

80erential-equations-variable-stepsize-and-transformation-approach

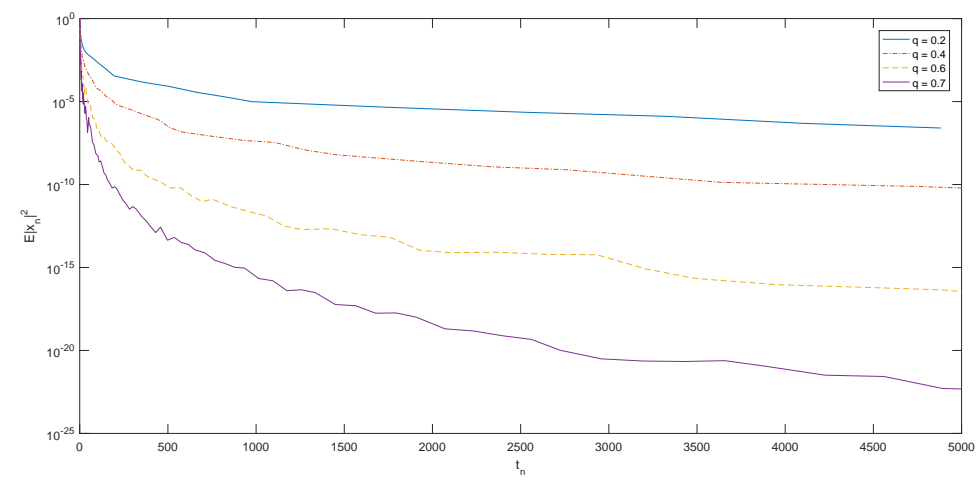



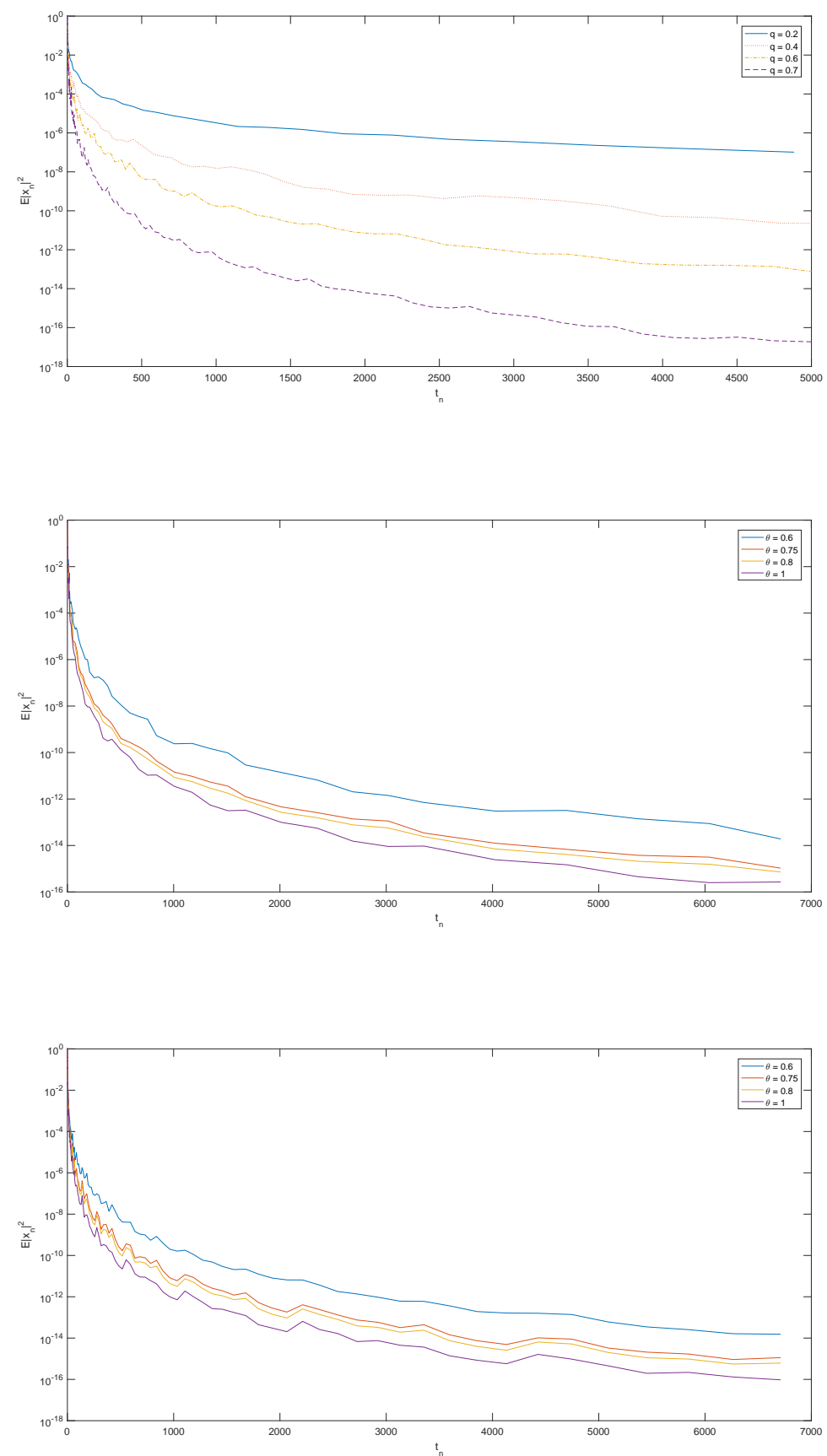